\title{
Trajectory tracking control of a quadrotor via fuzzy terminal sliding mode controller in the presence of uncertainty
}

\author{
Bahare Khalili' ${ }^{1}$, Mohammad Rezaei ${ }^{2}$ \\ ${ }^{1}$ MS student, Malek Ashtar University \\ ${ }^{2}$ Assistant Professor, Malek Ashtar University
}

\begin{abstract}
The purpose of this research is to design a robust controller in the presence of noise and uncertainty for trajectory tracking of a quadrotor flying robot. This paper presents a new method in which fuzzy logic controller is used to determine the optimal controller coefficients of a high order sliding mode controller. This method improves control behaviors against parametric uncertainty for a quadrotor trajectory tracking control. Results of simulation in two cases with parametric uncertainty and without uncertainty for trajectory tracking control demonstrate robustness and good performance of the proposed controller against uncertainty.
\end{abstract}

Keywords: Quadrotor, Fuzzy terminal sliding mode controller, Trajectory tracking, Uncertainty

\section{Introduction}

Quadrotors are used for conducting military missions that are boring or dangerous for manned aerial vehicles [1] and in cases, such as the control and supervision of the battleground, border patrol, public security monitoring, etc. [2]. Moreover, those used in the civilian applications of the aerial mapping and forest fire detection.

Quadrotor dynamics model with four thrust that is generated by four motors is a 6DOF* model. This topic faces the control issue with complexity. In addition, adding uncertainty to the system dynamics model creates more challenges for controller design

[3].

Several controllers have been designed for the control of position tracking, trajectory tracking and attitude stabilization of quadrotors, Including the sliding mode control [4], fuzzy control [5], robust adaptive control [6] and optimal control [7].

The robust sliding mode controller with fuzzy tuning is used for linear systems. One of the methods to decrease the chattering of sliding mode control without strong performance risk is using fuzzy logic control for adjusting coefficients [8].

Trajectory tracking control of the robot via a fuzzy sliding mode controller is investigated in [9]. Fuzzy sliding mode control is used for $\mathrm{SISO}^{\dagger}$ nonlinear systems [10].

Due to the increased applications of these UAV ${ }^{\ddagger}$, as well as the sensitivity of different mission systems to noise, disturbance and uncertainties, design of a robust control against these agents is important. In this paper, we discuss about a robust

\footnotetext{
* Degree of freedom

${ }^{\dagger}$ Single-input single-output

¥Unmanned aerial vehicle
} 
controller design in the presence of noise and uncertainties which maintains the stability of the system.

Discussions presented in this paper include quadrotor modeling, trajectory tracking controller design, fuzzy logic controller design, simulation and conclusions.

\section{Quadrotor modeling}

Dynamic model of the quadrotor is given by [11]-[13]

$x=f(x)+\ddot{g}(x) u+d(t)$

where $f(x) \in R^{n \times 1}$ is an unknown nonlinear vector function; $g(x) \in R^{n \times n}$ is an unspecified matrix; $u(t) \in R^{n \times 1}$ is vector of control and $d(t) \in R^{n \times 1}$ is an unknown nonlinear distortion. In (1) state vector $x(t) \in R^{n \times 1}$ is defined as follows [14].

$x \stackrel{\text { deff }}{=}\left[Z_{1} \phi \theta \psi\right]$

where $Z_{1} \in R$ is height; $\phi, \theta, \psi \in R$ respectively indicates the roll angle, pitch angle, yaw angle. $f(x)$ and $g(x)$ in (1) are defined as [14]

$$
f(x)=\left[\begin{array}{c}
-g \\
\frac{-\dot{\theta} \dot{\psi}\left(J_{z}-J_{y}\right)}{J x} \\
\frac{-\phi \dot{\psi}\left(J_{x}-J_{z}\right)}{J_{y}} \\
\frac{-\dot{\theta} \dot{\phi}\left(J_{y}-J_{x}\right)}{J_{z}}
\end{array}\right], \quad g(x)=\left[\begin{array}{cccc}
\frac{\cos \theta \cos \phi}{m} & 0 & 0 & 0 \\
0 & \frac{l}{J_{x}} & 0 & 0 \\
0 & 0 & \frac{l}{J_{y}} & 0 \\
0 & 0 & 0 & \frac{l}{J_{z}}
\end{array}\right]
$$

where $m \in R$ is variable mass of quadrotor; $l[\mathrm{~m}]$ is distance of motor and center of quadrotor. $I_{x} J_{y^{n}} J_{z} \in R$ are moments of inertia of the rigid body.

In this modeling, we have the following assumptions

$$
\begin{array}{ll}
-\frac{\pi}{2} \leq \phi(t) \leq \frac{\pi}{2}, & -\frac{\pi}{2} \leq \theta(t) \leq \frac{\pi}{2} \\
\|d(t)\| \leq \varepsilon_{1}, & \|d(t)\| \leq \varepsilon_{2},
\end{array}
$$

(5) expresses that disturbance is sufficiently smooth and limited. $\varepsilon_{i}$ is limited constant for $t=1,2,3$. Disturbance should be limited, otherwise the system (1) is out of control. 


\section{Trajectory tracking control design}

In this section, the continuous robust control technique is presented. This technique results a quadrotor tracking of trajectory in the presence of parametric uncertainty in the state matrix of input variables and immeasurable, nonlinear and unknown disturbance. Further development is based on the assumption that $x(t)$ and $\dot{x}(t)$ are measurable. The structure of higher order sliding mode controller has been developed to compensate the disturbance and increasing uncertainty, exploiting the least information of system and estimation of the unknown feed forward input matrix.

The goal is to design a robust tracking control which guarantees track of the desired time-varying trajectory $x_{d}(t) \in R^{n \times 1}$ with unknown disturbance and uncertainty in dynamic model.

By setting this goal, position tracking error $e_{1}$ is shown as

$$
e_{1} \triangleq x-x_{d}
$$

To simplify the controller design and equations, $\boldsymbol{e}_{2}(t)$ and $r(t)$ will be shown as below

$e_{2} \stackrel{\text { Hef }}{=} e_{1}+\alpha_{1} e_{1}, \quad r \stackrel{\text { gef }}{=} e_{2}+\alpha_{2} e_{2}$

where $\alpha_{1}$ and $\alpha_{2}$ are the control constant and positive gains. Based on (7)

$e_{1}(t) \rightarrow 0 \Rightarrow e_{1}(t) \rightarrow 0 \Rightarrow r(t) \rightarrow 0$

So, the aim of the controller can be posed as

$$
\left\|e_{1}(t)\right\| \rightarrow 0
$$

Dynamics of open loop tracking error can be developed by taking the time derivative of $r(t)$ and using (1), (4), (6)

$$
\begin{aligned}
\dot{r}(t) & =f(x)-f\left(x_{d}\right)+\alpha_{1} \ddot{e}_{1}+\dot{g}(x) u+g(x) \dot{u} \\
& +\alpha_{2} \dot{e}_{2}+f\left(x_{d}\right)+d(t)-\ddot{x}_{d}(t)
\end{aligned}
$$

It is assumed that the desired path $x_{d}(t)$ is limited. This means

$$
\left\|x_{d}(t)\right\|,\left\|\dot{x}_{d}(t)\right\|,\left\|\ddot{x}_{d}(t)\right\| \leq \varepsilon_{T}
$$

where $\varepsilon_{T} \in R$ is a limited, positive and defined constant.

Multiplication of control input by a variable state matrix including parametric uncertainty is a challenge in controller design. To compensate the uncertainty, the matrix $g(x)$ is separated to known terms. Nominal matrix $g_{0}(x) \in R^{n \times m}$ and constant matrix of certainty $\Delta g \in R^{n \times n}$ will be as follows

$$
g(x)=g_{0}(x) \Delta g, \quad 1-\varepsilon \leq\|\Delta g\|_{\infty} \leq 1+\varepsilon
$$


where $\varepsilon \in(0,1)$ is a limited, defined and positive constant, $\|\Delta g\|_{\infty}$ is infinite induced norm of the matrix. Uncertainties constant matrix $\Delta g$ is a diagonal matrix that shows parametric indeterminacy of $J_{x}, J_{y}, J_{z}$, and $m, l$.

by inserting (9) in (8)

$$
\dot{r}=N_{\dot{u}}+\widetilde{N}+\Delta g\left(\dot{g}_{0}(x) u+g_{0}(x) \dot{u}\right)-e_{2}
$$

where $N_{d}$ and $\tilde{N}$ are auxiliary functions and are given by

$$
N_{d} \stackrel{d e f}{=} d(\mathrm{t})+f\left(x_{d}\right)-\ddot{x}_{d}
$$

$$
\widetilde{N} \stackrel{\text { anf }}{=} f(x)-f\left(x_{d}\right)+\alpha_{1} \ddot{e}_{1}+\alpha_{2} \dot{e}_{2}+e_{2}
$$

The values of $N_{d}, \tilde{N}$ and time derivative of $N_{d}$ are as follows [15], [16]

$$
\|\tilde{N}\| \leq \rho(\|Z\|)\|Z\|, \quad\left\|N_{d}\right\| \leq \varepsilon_{N_{d},} \quad \dot{N}_{d} \leq \varepsilon_{N_{d}}
$$

where $\varepsilon_{N_{i d}}, \varepsilon_{N_{i}} \in R$ are limited, defined and positive constants and $z(t)$

$$
z(t) \stackrel{\text { ㅆsff }}{=}\left[\begin{array}{lll}
e_{1}^{T} & e_{2}^{T} & r^{T}
\end{array}\right]^{T}
$$

$\rho(0) \in R$ is non-decreasing, inverse, global and positive [12]. Based on (11) and stability analysis, so control input can be designed as

$$
\dot{u}=-g_{0}(x)^{-1}\left(\beta \operatorname{sgn}\left(e_{2}\right)+\left(k_{1}+1\right) r+g_{0}(x) 1\right.
$$

$\beta, k_{1} \in R$ are invariant and affirmative gains of controller.

\section{Fuzzy terminal system design}

In fuzzy terminal method, we use fuzzy logic to determine gains of control with purpose of control behavior improvement. Implemented Fuzzy logic controller behaviors like two-input two-output systems are considered here. This means that error of system and its derivative are used as input with using optimal laws (that is achieved from trial and error under various quiescent conditions), the control gains of system are achieved. Figure 1 shows the fuzzy logic controller:

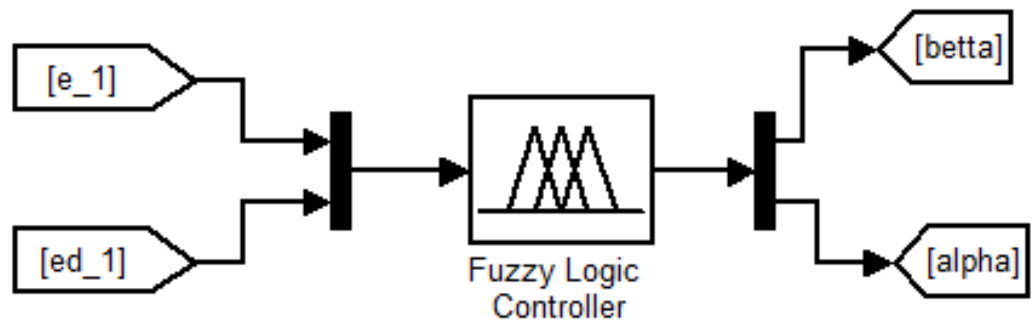


Figure 1: Fuzzy logic controller

Fuzzy logic is shown as below

Table 1: Fuzzy table

\begin{tabular}{|c|c|c|c|}
\hline Error & Negative & Zero & Positive \\
Derivative of error & S-S & S-M & M-M \\
\hline Negative & S-M & M-M & B-M \\
\hline Zero & M-M & B-M & B-B \\
\hline Positive & & & \\
\hline
\end{tabular}

\section{Simulation}

In this section, we discuss simulation results including control signals, trajectory tracking and tracking errors. Simulations were run based on the parametric and numerical values in MATLAB software to evaluate the performance of the controller.

The system model used in simulation is based on (1) to (3) and the used assumptions are according to (4) and (5) we have $f(x) \in R^{4 \times 1}, g(x) \in R^{4 \times 4}, u(t) \in R^{4 \times 1}$ and $d(t) \in R^{4 \times 1}$.

Parameters used in system are according to real model. These parameters are exerted in practical experiments and those have been referred to.

Maximum system's trust range has been assumed based on the practical experiments. Each motor of the system has a stable range up to 38 newton $[\mathrm{N}]$ which causes the maximum thrust range of the system to be around 125 .

The certainty matrix $\Delta g$ is defined as

$$
\Delta g=\left[\begin{array}{cccc}
b_{1} & 0 & 0 & 0 \\
0 & b_{2} & 0 & 0 \\
0 & 0 & b_{3} & 0 \\
0 & 0 & 0 & b_{4}
\end{array}\right]
$$

Nonlinear disturbance $d(t)$ demonstrated in (1) represents a model to simulate the noise in the system. It will be assumed as a Gaussian distribution: 


$$
d(t)=\left[\begin{array}{c}
N(0,1) \\
N(0,0.5) \\
N(0,0.5) \\
N(0,0.5)
\end{array}\right]
$$

Continuous and differentiable desired trajectory emphasizes tracking ability of the system as shown by the following equation

$$
x_{d}(t)=\left[\begin{array}{c}
0.1 t+6 \\
\frac{20 \pi}{180} \sin \left(\frac{t}{10}\right) \\
20 \pi \\
\frac{180}{18} \sin \left(\frac{t}{10}+\frac{\pi}{3}\right) \\
\frac{20 \pi}{180} \sin \left(\frac{t}{10}+\frac{\pi}{6}\right)
\end{array}\right]
$$

Control parameters are selected so that controller will have the best and optimal performance (in terms of control energy consumption). Controller parameters in simulation were obtained using presented fuzzy logic.

Values of uncertainty matrix $\Delta g$ are respectively

$$
b_{1}=0.8, b_{2}=0.5, b_{3}=0.7, b_{4}=0.6
$$

Under the efficacy of the uncertainty parameters, mass and moment inertia physical parameters from $\% 20$ to $\% 50$ will be indefinite. Moreover, Gaussian noise with powers from 0.5 to 1 will affect each of the system dynamics.

Simulations are presented in two cases, 1) without uncertainty, 2) with $\% 50$ uncertainty which is the most severe type of uncertainties for the robot.

In addition, there is noise in the system with supposed powers. The purpose of this section is to emphasize the robustness of the controller in the presence of noise and uncertainty. Figures 2 and 3, show the applied control signals to the system. 

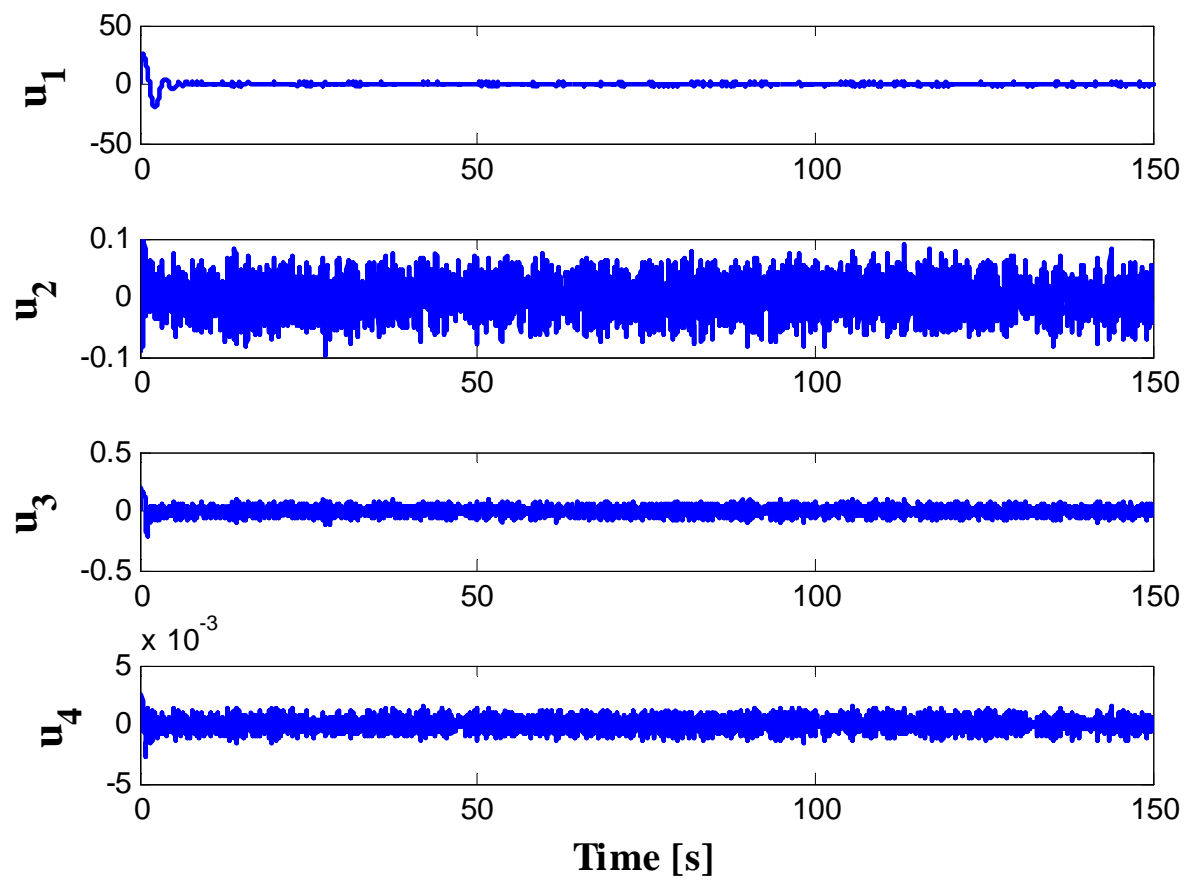

Figure 2: Control signals of the system without uncertainty.
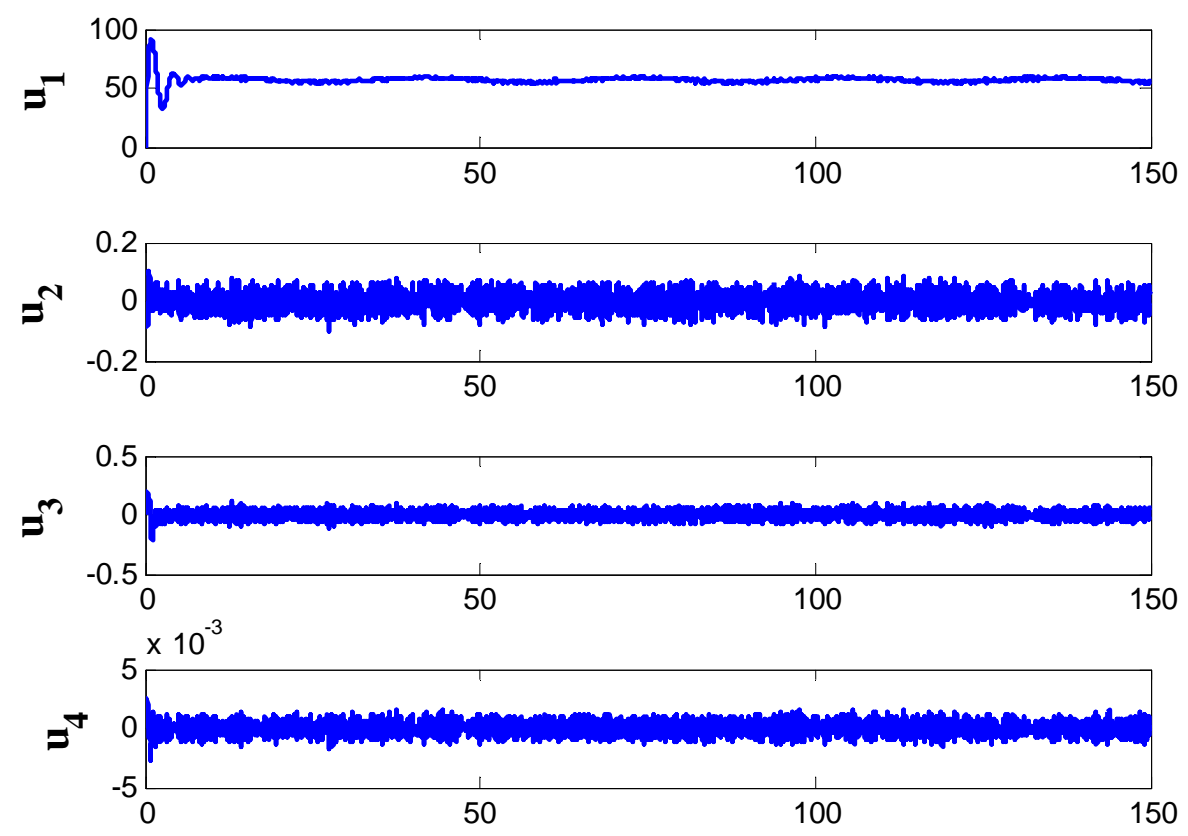

Time [s]

Figure 3: Control signals of the system with uncertainty. 
Control signals due to existence noise in the system states are noisy. Obviously, noise reduction or removal will pave the control's forces.

In the following Figures 4 to 7 show waveforms of output and the errors of each output variable. Then controller performance is checked.
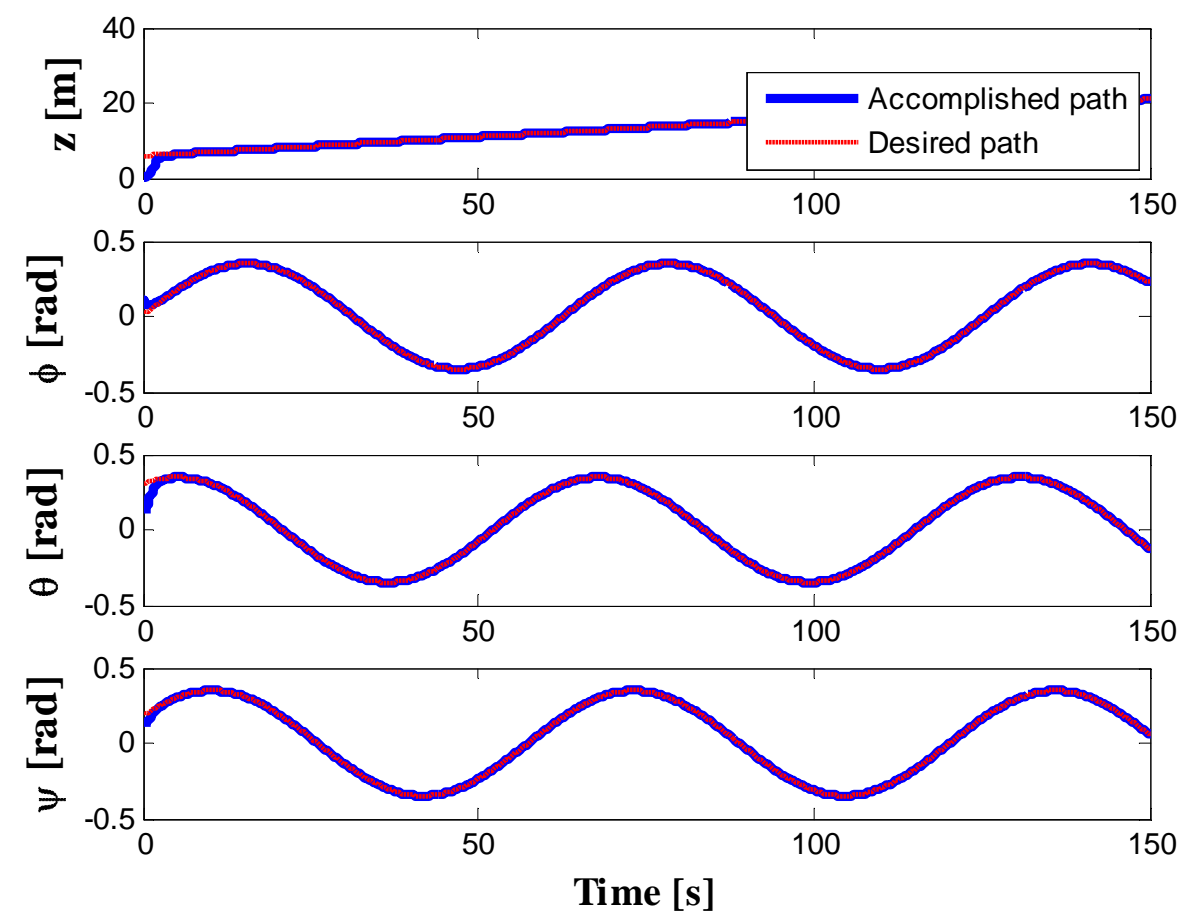

Figure 4: Trajectory tracking of the system without uncertainty.
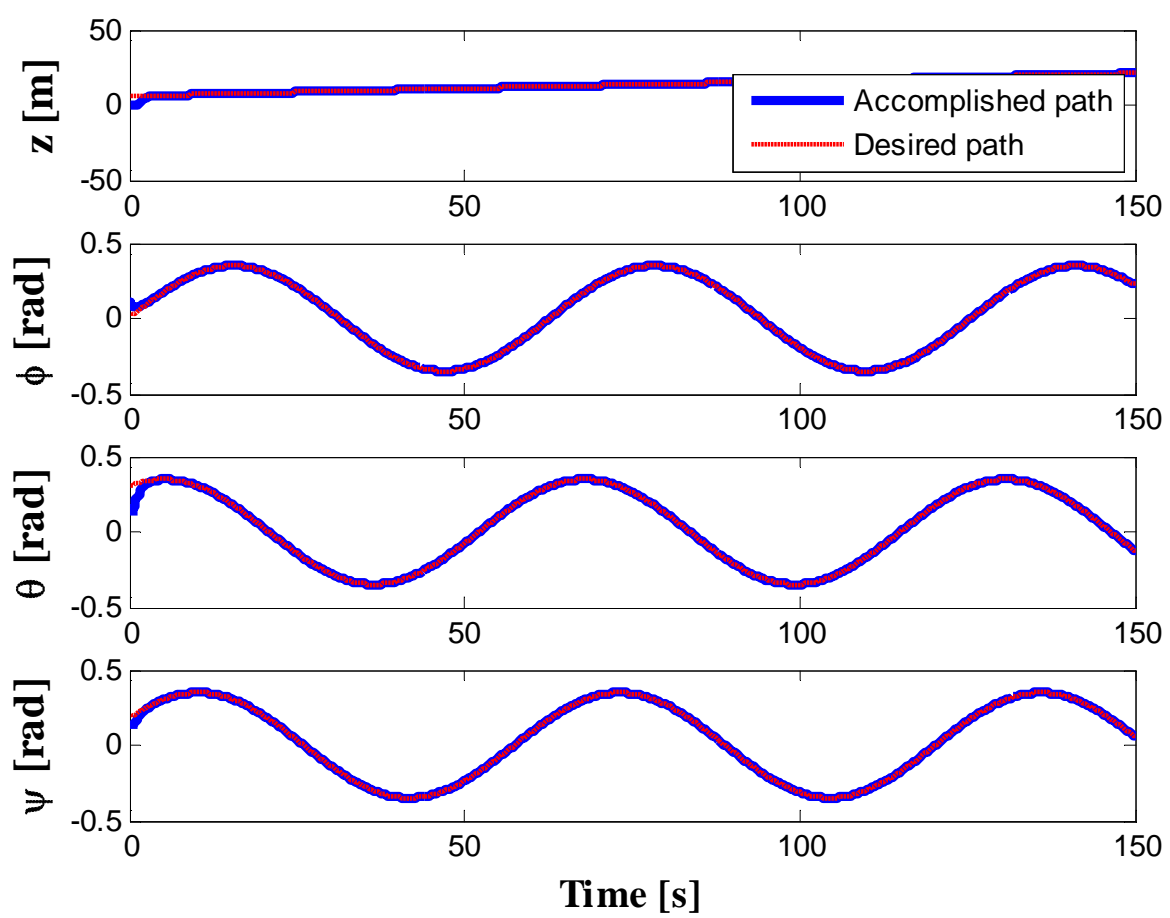

Figure 5: Trajectory tracking of the system with uncertainty. 
Bulletin de la Société Royale des Sciences de Liège, Vol. 85, 2016, p. 227 - 237

Figures 4, 5 show the accomplished path next to the desired path. Based on the (1) to (5) when uncertainty is applied, the dynamic of system will change extremely. Therefore, the existence of robust controller is necessary for system stabilization.
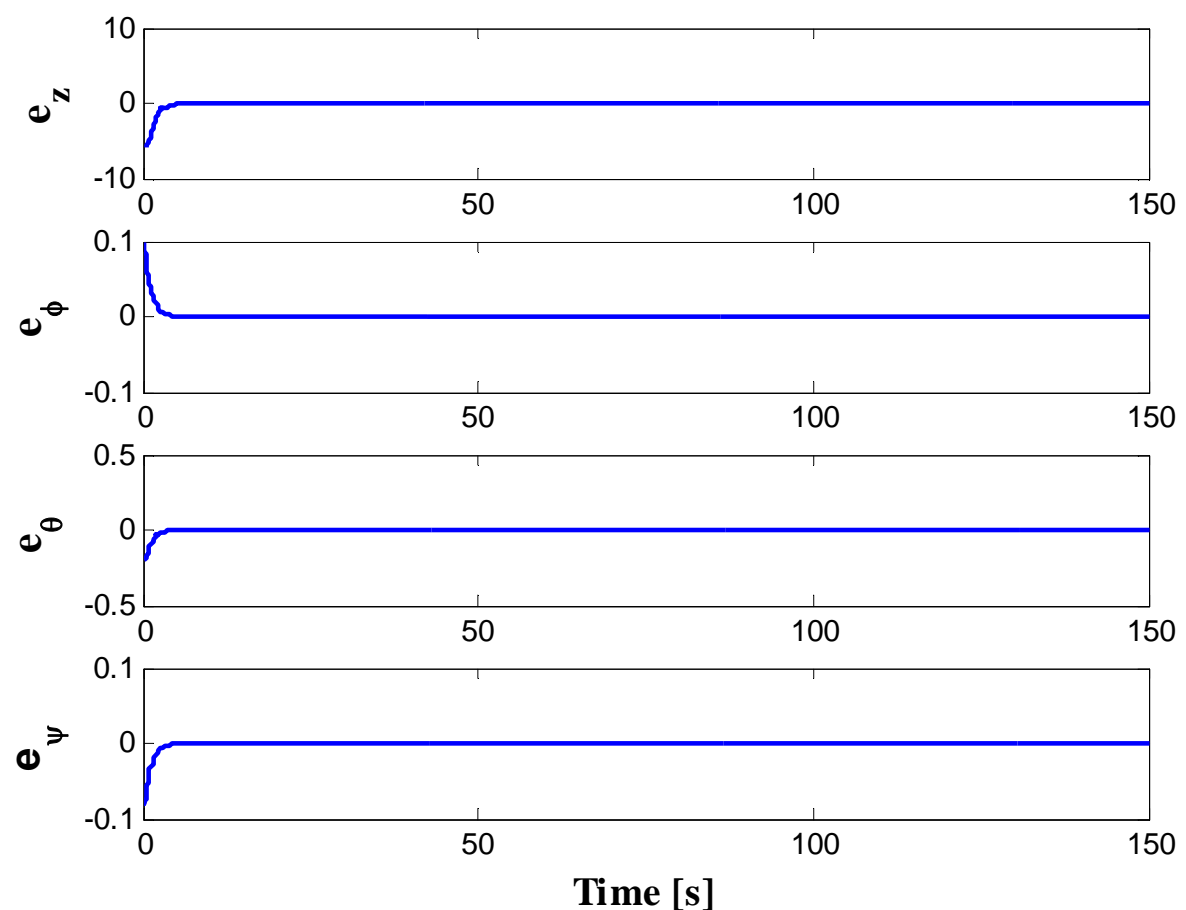

Figure 6: Tracking errors of the system without uncertainty.
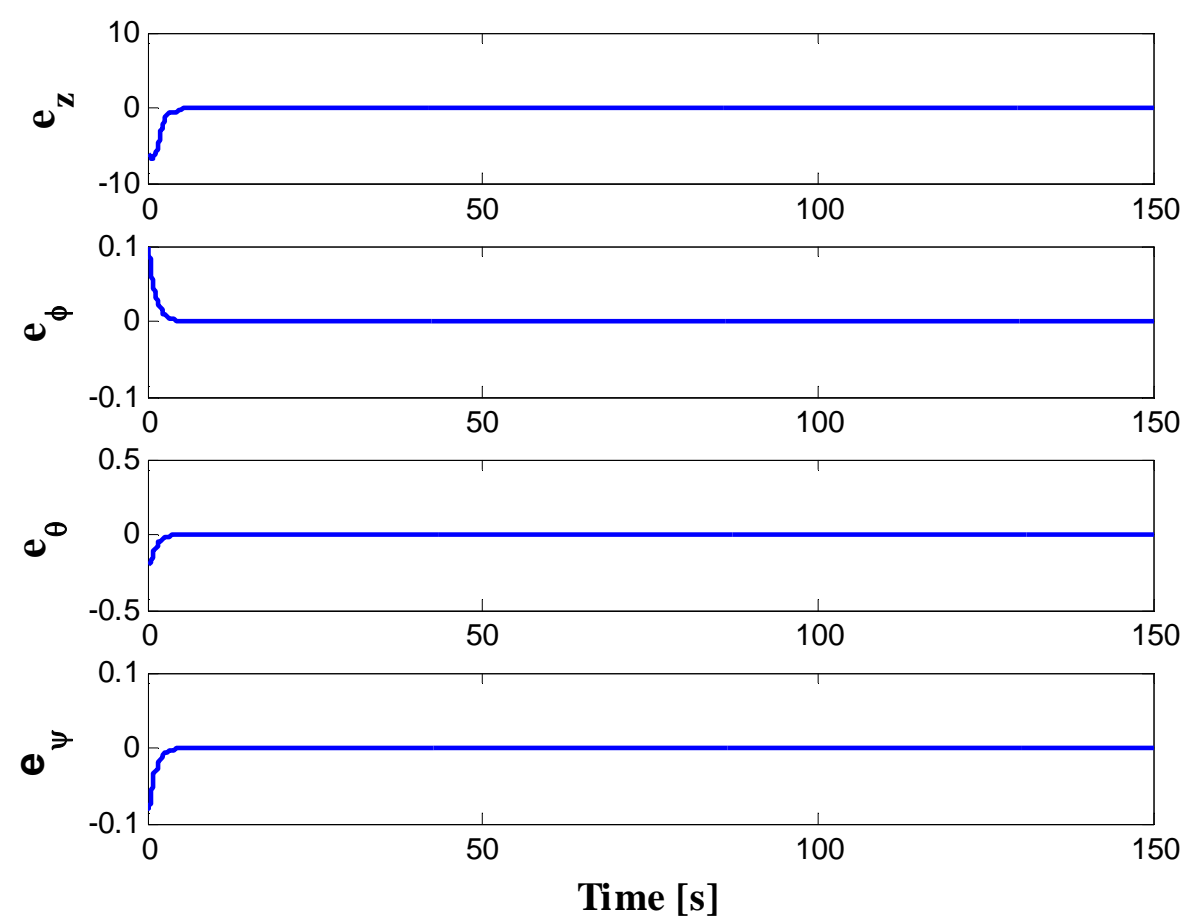

Figure 7: Tracking errors of the system with uncertainty. 
It has to be considered when the system is without uncertainty, for 4 output variables settling time is less than 2 seconds and steady state error is zero, showing the optimal performance of sliding mode controller for the system.

After applying uncertainty, except settling time that increased 1 [s] second, other control factors such as steady state error still remain in this case. The reason of increasing settling time is the presence of uncertainty. Removing it in the first moments extends the process.

\section{Conclusions}

This paper displays good performance of fuzzy terminal sliding mode controller for a quadrotor in the presence of noise and uncertainty. Bets optimal performance achieved in terms of control energy consumption. The controller can be used for real applications and environments where optimal energy consumption is needed. The results of simulation demonstrate the applied controller can control \%50 of certainties and create stability in the system.

\section{Future works}

In future works, we will consider six degree of freedom of quadrotor flying robot and control the system completely. Next step is to design and implement the control of our robot in robotic research laboratory.

\section{References}

[1] M. Wierema, "Design, Implementation and Flight Test of Indoor Navigation and Control System for a Quadrotor UAV,” 2008.

[2] D. Luo, W. Xu, and S. Wu, "UAV Formation Flight Control and Formation Switch Strategy," Comput. Sci. Educ. (ICCSE), 2013 8th Int. Conf. on. IEEE, pp. 264-269, 2013.

[3] W. Sulistyono and M. Lawley, "Robust stabilization of a quadrotor aerial vehicle in presence of sensor failures," vol. 2, no. May, pp. 1-6, 2002.

[4] L. Besnard, Y. B. Shtessel, and B. Landrum, "Quadrotor vehicle control via sliding mode controller driven by sliding mode disturbance observer," J. Franklin Inst., vol. 349, no. 2, pp. 658-684, 2012.

[5] A. Prayitno, V. Indrawati, and G. Utomo, "Trajectory Tracking of AR.Drone Quadrotor Using Fuzzy Logic Controller," TELKOMNIKA (Telecommunication Comput. Electron. Control., vol. 12, no. 4, p. 819, 2014.

[6] K. S. Yang and C. C. Cheng, "Robust Adaptive Controller Design for a Quadrotor Helicopter," AMM, vol. 284-287, pp. 2296-2300, 2013. 
[7] R. Zawiski and M. Błachuta, "Modelling and optimal control system design for quadrotor platform - an extended approach," Bull. Polish Acad. Sci. Tech. Sci., vol. 62 , no. $3,2014$.

[8] Q. P. Ha, "Robust sliding mode controller with fuzzy tuning," Electron. Lett., vol. 32, no. 17, pp. 1626-1628, 1996.

[9] H. Mei, "Trajectory Tracking Control of Robot via a Fuzzy Sliding-Mode Controller," AMR, vol. 433-440, pp. 4154-4158, 2012.

[10] W. Chang, J. B. Park, Y. H. Joo, and G. Chen, "Design of robust fuzzy-modelbased controller with sliding mode control for SISO nonlinear systems," Fuzzy Sets Syst., vol. 125, no. 1, pp. 1-22, 2002.

[11] Z. Dydek, A. Annaswamy, and E. Lavretsky, "Adaptive Control of Quadrotor UAVs in the Presence of Actuator Uncertainties," in AIAA Infotech@Aerospace 2010 Atlanta, Georgia, 2010, pp. 20-22.

[12] A. Chamseddine, Y. Zhang, C.-A. Rabbath, J. Apkarian, and C. Fulford, "Model Reference Adaptive Fault Tolerant Control of a Quadrotor UAV," in Infotech@Aerospace 2011 St. Louis, Missouri, 2011, pp. 29-31.

[13] A. Chamseddine, Y. Zhang, and C.-A. Rabbath, "Adaptive Trajectory Planning for a Quad-rotor Unmanned Aerial Vehicle," in AIAA Guidance, Navigation, and Control Conference Toronto, Ontario, Canada, 2010, pp. 2-5.

[14] X. Zhang, Y. Zhang, C.-Y. Su, and Y. Feng, "Fault-Tolerant Control for Quadrotor UAV via Backstepping Approach," in 48th AIAA Aerospace Sciences Meeting Including the New Horizons Forum and Aerospace Exposition Orlando, Florida, 2010, pp. 4-7.

[15] B. Xian, D. M. Dawson, M. S. de Queiroz, and J. Chen, "A continuous asymptotic tracking control strategy for uncertain multi-input nonlinear systems," in Proceedings of the 2003 IEEE International Symposium on Intelligent Control ISIC-03, 2003, pp. 52-57.

[16] P. M. Patre, W. MacKunis, C. Makkar, and W. E. Dixon, "Asymptotic Tracking for Systems with Structured and Unstructured Uncertainties," in Proceedings of the 45th IEEE Conference on Decision and Control, 2006, pp. 441-446. 